\title{
Flat band induced by the interplay of synthetic magnetic flux and non-Hermiticity
}

\author{
L. Jin* \\ School of Physics, Nankai University, Tianjin 300071, China
}

(Received 29 October 2018; revised manuscript received 16 January 2019; published 6 March 2019)

\begin{abstract}
A flat band is nondispersive and formed under destructive interference. Although flat bands are found in various Hermitian systems, to realize a flat band in non-Hermitian systems is an interesting task. Here, we propose a flat band in a parity-time symmetric non-Hermitian lattice. The proposed flat band has entirely real energy and is formed at an appropriate match between synthetic magnetic flux and non-Hermiticity. The flat band energy is tunable. At a weak intercell coupling, the flat band is isolated, whereas at a strong intercell coupling, it intersects with the dispersive band at the non-Hermitian phase transition point. The eigenstates of the flat band are compact localized states and are confined in one, two, or three unit cells at the edges or inside the non-Hermitian lattice.
\end{abstract}

DOI: 10.1103/PhysRevA.99.033810

\section{INTRODUCTION}

A flat band is entirely constituted by degenerate states, is nondispersive, and has a zero group velocity. The superposition of nondispersive flat band modes propagates without diffraction. A flat band exhibits many peculiar features of wave propagation [1], localization length scaling [2,3], and unconventional Anderson localization [4]. A simple model with a flat band is the Lieb lattice: a zero-energy flat band intersects two linearly dispersive bands at the Dirac point [5]. The Lieb lattice is a line-centered square lattice that can be realized in photonics by trapping ions in optical lattices [6] or through the laser writing technology in optical waveguides [7-9]. In addition, optical lattices in other geometric structures of diamond (rhombic) [2,10,11], kagome [12,13], honeycomb $[14,15]$, pyrochlore [16,17], and dice $\left(\mathcal{T}_{3}\right)[18,19]$ lattices support flat bands $[20,21]$.

A magnetic flux renders flat bands without disorders; thus particles and light can be perfectly trapped in a compact localization, following the trapping mechanism of destructive interference. This mechanism differs from the trapping mechanism in a spatially localized state induced by disorders [22]. It has been proposed that the Aharonov-Bohm (AB) cage is induced by magnetic flux in a two-dimensional dice lattice [18] and a quasi-one-dimensional (quasi-1D) diamond lattice $[10,23,24]$. The $A B$ cage has been experimentally observed in quasi-1D coupled waveguide lattices [25-27].

Parity-time $(\mathcal{P} \mathcal{T})$ symmetric non-Hermitian systems are manifested in various microwave, optical, acoustic, and electronic systems [28-54]. They have potential applications in nonreciprocal dynamics [55-58], topological energy transfer

\footnotetext{
*jinliang@nankai.edu.cn

Published by the American Physical Society under the terms of the Creative Commons Attribution 4.0 International license. Further distribution of this work must maintain attribution to the author(s) and the published article's title, journal citation, and DOI.
}

[59,60], and novel lasing [61-64]. Recently, investigations on flat bands have been extended to $\mathcal{P} \mathcal{T}$-symmetric nonHermitian systems [65-70]. It is shown that the Lieb stripe maintains a flat band regardless of the non-Hermiticity of $\mathcal{P} \mathcal{T}$ symmetric gain and loss [65]. $\mathcal{P} \mathcal{T}$-symmetric gain and loss perturbation lifts the degeneracy of a flat band in a stub lattice [66]. A flat band can be formed by non-Hermiticity at the $\mathcal{P} \mathcal{T}$ symmetric phase transition point in a triangular lattice [67] and a cross-stitch lattice [69], and a polynomial increase of the intensity for a single site excitation has been revealed $[68,69]$. In addition, another type of zero-energy flat band with net amplification and attenuation emerging from lattices under nonHermitian particle-hole symmetry has been investigated [70].

In this paper, we report a configuration of a quasi-1D $\mathcal{P} \mathcal{T}$-symmetric non-Hermitian lattice. The lattice unit cell comprises a $\mathcal{P} \mathcal{T}$-symmetric non-Hermitian triangular ring enclosed synthetic magnetic flux, which is cross-stitch coupled along the translationally invariant direction. A realenergy flat band forms under destructive interference at an appropriate match between synthetic magnetic flux and nonHermiticity. The energy of the flat band is enforced to be zero by chiral symmetry; the intracell coupling between the active and dissipative lattices breaks chiral symmetry, thus enabling tuning of the flat band energy. The flat band can be isolated or intersected with dispersive bands, where the band intersections are exceptional points (EPs). At EPs, the degree of non-Hermiticity corresponds to the $\mathcal{P} \mathcal{T}$-symmetric phase transition threshold of the lattice. The flat band consists of compact localized states (CLSs), which at the band intersections are the bound states in the continuum. CLSs are confined in the single unit cell of the system in the absence of chiral symmetry; by contrast, zero-energy flat band CLSs can distribute in two or three unit cells.

The paper is organized as follows. In Sec. II, a quasi-1D $\mathcal{P T}$-symmetric non-Hermitian system is modeled. In Sec. III, the energy-band structure of the non-Hermitian system is studied. The conditions for forming a flat band, $\mathcal{P} \mathcal{T}$ symmetric phase transition, and energy-band structures are also explored. In Sec. IV, the localized modes and edge 


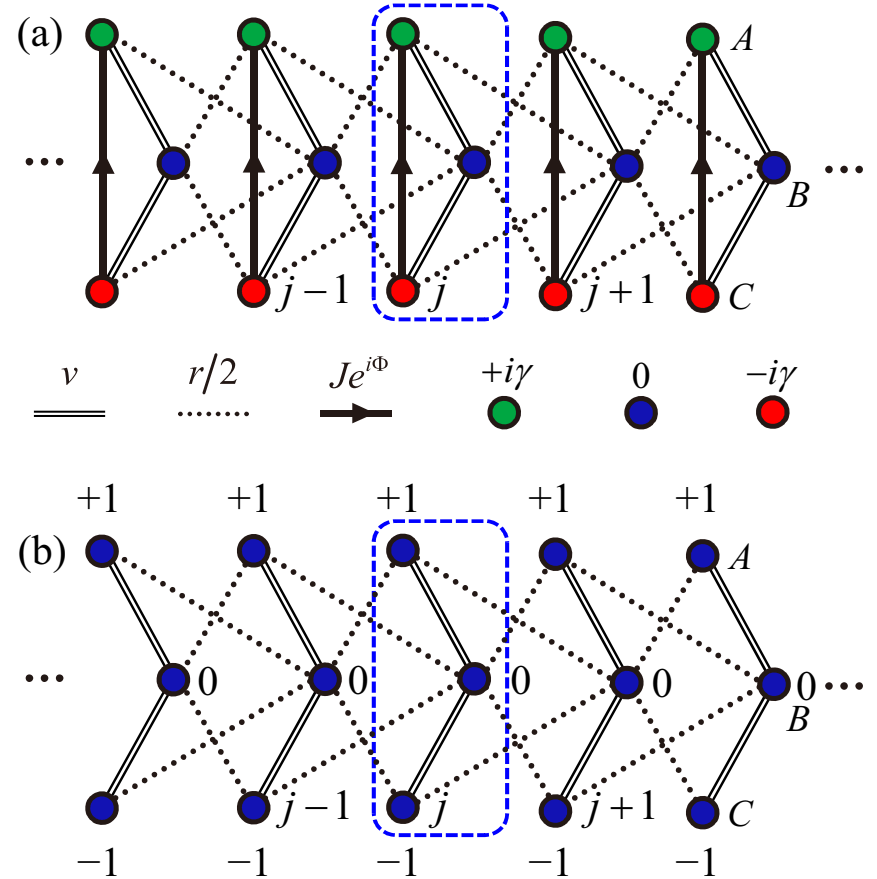

FIG. 1. Schematics of (a) a $\mathcal{P} \mathcal{T}$-symmetric non-Hermitian lattice and (b) a Hermitian cross-stitch lattice. Sublattice $A(C)$ with gain (loss) is depicted in green (red), whereas the passive sublattice $(B)$ is depicted in blue. The coupling between sublattices $A$ and $C$ is asymmetric; the arrows denote the phase direction. The dashed blue rectangle indicates the lattice unit cell. The dotted black lines represent the cross-stitch intercell coupling between the sublattices. A compact localized state of antisymmetric excitation $(1,0,-1)$ is shown in (b).

modes of the flat band are shown. In Sec. V, the results are summarized and the prospects are presented.

\section{II. $\mathcal{P} \mathcal{T}$-SYMMETRIC NON-HERMITIAN LATTICE}

The non-Hermitian lattice considered in this study is schematically illustrated in Fig. 1(a). Three types of sublattices $A, B$, and $C$ are active, passive, and dissipative, respectively; the gain and loss rates are $\gamma[33,37,42] . A_{j}, B_{j}$, and $C_{j}$ constitute the $j$ th unit cell (denoted by the dashed blue rectangle). The system is $\mathcal{P} \mathcal{T}$ symmetric with respect to the parity operation $\mathcal{P} A_{j} \mathcal{P}^{-1}=C_{N+1-j}, \mathcal{P} B_{j} \mathcal{P}^{-1}=B_{N+1-j}$ and the time-reversal operation $\mathcal{T} i \mathcal{T}^{-1}=-i . N$ is the total site number of each sublattice.

The tight-binding Hamiltonian is given by

$$
\begin{aligned}
H= & \sum_{j=1}^{N}\left[\frac{r}{2}\left(a_{j}^{\dagger} b_{j-1}+a_{j}^{\dagger} b_{j+1}+c_{j}^{\dagger} b_{j-1}+c_{j}^{\dagger} b_{j+1}\right)\right. \\
& \left.+J e^{i \Phi} a_{j}^{\dagger} c_{j}+v\left(c_{j}^{\dagger} b_{j}+b_{j}^{\dagger} a_{j}\right)+\text { H.c. }\right] \\
& +i \gamma\left(a_{j}^{\dagger} a_{j}-c_{j}^{\dagger} c_{j}\right),
\end{aligned}
$$

where $a_{j}^{\dagger}\left(a_{j}\right), b_{j}^{\dagger}\left(b_{j}\right)$, and $c_{j}^{\dagger}\left(c_{j}\right)$ are the creation (annihilation) operators that satisfy the periodical boundary condition $a_{N+1}=a_{1}, b_{N+1}=b_{1}$, and $c_{N+1}=c_{1}$, respectively.
The system parameters $(\gamma, v, J, r)$ are all considered to be positive real values without loss of generality. The cross-stitch intercell coupling strength is $r / 2(r \neq 0)$ [71-73]. The passive sublattice couples to the active and dissipative sublattices with strength $v$. The asymmetric coupling with the nonreciprocal phase factor $J e^{ \pm i \Phi}$ between the active and dissipative sublattices induces a gauge-invariant synthetic magnetic flux in each triangular unit cell. A nonreciprocal phase factor can be realized in optical systems through chiral-light interaction [74], dynamical modulation [75], photon-phonon interaction [76], and optical path length imbalance [77] in coupled waveguides and resonators. This factor can also be realized through laser-induced tunneling [78] and shaking of the lattice or Floquet engineering [79] for cold atoms in optical lattices [80]. Moreover, synthetic magnetic flux has been realized beyond optics in microwave [81] and acoustic [82] regimes.

By applying a Fourier transformation $a_{k}=$ $N^{-1 / 2} \sum_{j=1}^{N} e^{i k j} a_{j}, \quad b_{k}=N^{-1 / 2} \sum_{j=1}^{N} e^{i k j} b_{j}, \quad$ and $c_{k}=$ $N^{-1 / 2} \sum_{j=1}^{N} e^{i k j} c_{j}$, where the wave vector $k=2 \pi n / N$ (integer $n \in[1, N]$ ) and the Hamiltonian in the momentum space is rewritten as $H=\sum_{k} H_{k}$, where $H_{k}$ is given by

$$
H_{k}=\left(\begin{array}{ccc}
i \gamma & v+r \cos k & J e^{i \Phi} \\
v+r \cos k & 0 & v+r \cos k \\
J e^{-i \Phi} & v+r \cos k & -i \gamma
\end{array}\right) .
$$

The $3 \times 3$ matrix $H_{k}$ is $\mathcal{P} \mathcal{T}$ symmetric under the definition of the parity operator

$$
\mathcal{P}=\left(\begin{array}{lll}
0 & 0 & 1 \\
0 & 1 & 0 \\
1 & 0 & 0
\end{array}\right)
$$

and the time-reversal operation $\mathcal{T} i \mathcal{T}^{-1}=-i$. Then, the Hamiltonian in the momentum space satisfies $(\mathcal{P} \mathcal{T}) H_{k}(\mathcal{P} \mathcal{T})^{-1}=H_{k}$. In addition, chiral symmetry exists when $J=0$ or the present synthetic magnetic flux is $\Phi=n \pi+\pi / 2(n \in \mathbb{Z})$, where $J e^{ \pm i \Phi}=0$ or $(-1)^{n}( \pm i J)$. Here, the chiral operator is defined as

$$
\mathcal{C}=\left(\begin{array}{ccc}
0 & 0 & 1 \\
0 & -1 & 0 \\
1 & 0 & 0
\end{array}\right)
$$

and the Hamiltonian in the momentum space satisfies $\mathrm{CH}_{k} \mathcal{C}^{-1}=-H_{k}$. In other conditions, chiral symmetry is absent. $H_{k}$ can possess a nonzero flat band through destructive interference without the confinement of chiral symmetry. $H_{k}$ has three energy bands, which can be analytically obtained through solving a cubic equation

$$
E_{k}^{3}-\left(2 s_{k}^{2}+J^{2}-\gamma^{2}\right) E_{k}-2 s_{k}^{2} J \cos \Phi=0,
$$

where $s_{k}=v+r \cos k$. The couplings $v, J$, and $r$ expand the energy bands; the energy bands shrink in the presence of gain and loss.

\section{FLAT BAND}

A flat band is nondispersive and independent of the momentum $k$. Destructive interference is crucial for the formation of a flat band, which can induce decoupling and isolation. To investigate the flat band in the non-Hermitian lattice, we 
start from a Hermitian situation of $J=\gamma=0$ to elucidate the formation of a flat band in a quasi-1D lattice. When $J=\gamma=0$, the system reduces to a cross-stitch lattice, as schematically illustrated in Fig. 1(b). If the cross-stitch coupling is zero $(r=0)$, the quasi-1D lattice possesses three flat bands because all the unit cells are disconnected. The isolated trimer in each unit cell has three eigenenergies zero and $\pm \sqrt{2} v$, where the eigenstate of the $j$ th unit cell for zero eigenenergy is $\left(A_{j}, B_{j}, C_{j}\right)=(1 / \sqrt{2}, 0,-1 / \sqrt{2})$; the vanishing distribution probability of the zero energy eigenstate on site $B_{j}$ indicates that antisymmetric excitation in sites $A_{j}$ and $C_{j}$ destructively interferes at site $B_{j}$.

The cross-stitch coupling connects the nearest-neighbor sites in the upper $(A)$ and lower $(C)$ sublattices with the central sublattice $(B)$. In the presence of cross-stitch coupling $(r \neq 0)$, antisymmetric excitation in sites $A_{j}$ and $C_{j}$ destructively interferes at sites $B_{j \pm 1}$; in addition, antisymmetric excitation in the unit cells $j \pm 1$ destructively interferes at site $B_{j}$. Thus the central sublattice is effectively decoupled with a vanishing distribution probability on the entire sublattice $B$, and a zeroenergy flat band is maintained in the presence of cross-stitch coupling.

Consider the non-Hermitian lattice with $J, \gamma \neq 0$ in Fig. 1(a). The analysis of cross-stitch lattice indicates that a flat band is formed when the sublattices $A$ and $C$ destructively interfere at the central sublattice $B$. In the following, we calculate the condition for maintaining such a destructive interference; that is the condition for the existence of a flat band in the non-Hermitian lattice. By acting $H_{k}$ [Eq. (2)] on $f_{k}=[1,0,-1]^{\mathrm{T}} / \sqrt{2}$, a Schrödinger equation $H_{k} f_{k}=E_{k} f_{k}$ is yielded, which gives

$$
H_{k}\left(\begin{array}{c}
1 \\
0 \\
-1
\end{array}\right)=\left(\begin{array}{c}
i \gamma-J e^{i \Phi} \\
0 \\
i \gamma+J e^{-i \Phi}
\end{array}\right)=E_{k}\left(\begin{array}{c}
1 \\
0 \\
-1
\end{array}\right),
$$

where $f_{k}=[1,0,-1]^{\mathrm{T}} / \sqrt{2}$ is an eigenstate of $H_{k}$ that requires

$$
i \gamma-J e^{i \Phi}=-\left(i \gamma+J e^{-i \Phi}\right),
$$

which gives $\gamma=J \sin \Phi$. Substituting $\gamma=J \sin \Phi$ back into the Schrödinger equation $H_{k} f_{k}=E_{k} f_{k}$, we obtain the eigenenergy $E_{k}=-J \cos \Phi$; which is independent of the momentum $k$. Therefore, a flat band is formed under the condition

$$
\gamma_{\mathrm{FB}}=J \sin \Phi .
$$

The flat band is induced by the interplay of non-Hermiticity and synthetic magnetic flux. Correspondingly, the eigenenergy of the flat band is

$$
E_{\mathrm{FB}}=-J \cos \Phi .
$$

Notably, the flat band energy is entirely real, and it is tunable through different matches between synthetic magnetic flux and non-Hermiticity, instead of pinning to zero energy in the Hermitian limit of the cross-stitch lattice at $J=\gamma=0$.

The condition given in Eq. (8) for the formation of the flat band can be alternatively obtained as follows. The crossstitch coupling of a lattice causes the dispersion in the energy bands; effectively decoupling sublattice $B$ under destructive interference enables the isolation of the unit cells, thereby
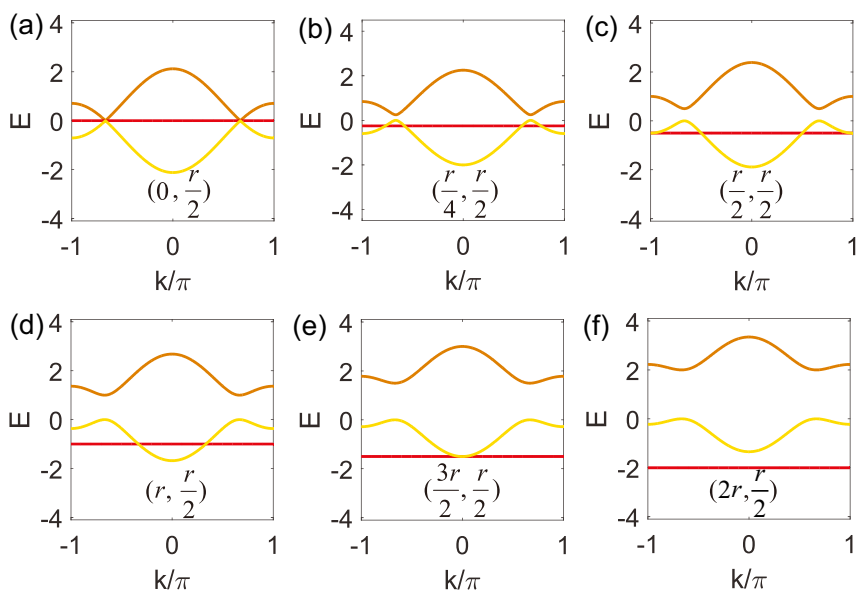

FIG. 2. Energy band structures with the flat band at different $(J|\cos \Phi|, v)$ values (marked at the bottom). All the energy bands are entirely real. The degree of non-Hermiticity is $\gamma=J \sin \Phi, \Phi=$ $\pi / 3$, and $r=1 . k=-\pi$, and $k=\pi$ represent an identical point.

forming the flat band. In the flat band, the triangular lattice in each unit cell effectively reduces into a $\mathcal{P} \mathcal{T}$-symmetric dimer with asymmetric coupling between sublattices $A$ and $C$ in the form of

$$
H_{\mathcal{P} \mathcal{T} \text { dimer }}=\left(\begin{array}{cc}
i \gamma & J e^{i \Phi} \\
J e^{-i \Phi} & -i \gamma
\end{array}\right) .
$$

The asymmetric coupling phase factor $e^{ \pm i \Phi}$ changes the eigenstates without varying the pair of eigenenergies $\pm \sqrt{J^{2}-\gamma^{2}}$. The destructive interference at the central sublattice $B$ requires antisymmetric excitation on sublattices $A$ and $C$, where we obtain identical constraint of Eq. (7) by acting $H_{\mathcal{P} \mathcal{T} \text { dimer on }}$ the antisymmetric state $[1,-1]^{\mathrm{T}} / \sqrt{2}$. Consequently, antisymmetric excitation is one of the two eigenstates of the $\mathcal{P} \mathcal{T}$ symmetric dimer, and the corresponding eigenenergy is $E_{\mathrm{FB}}$. Antisymmetric excitation is a cage solution confined in the single unit cell [10].

Any antisymmetric excitation of each unit cell is confined in the single unit cell; thus the eigenfunctions of the flat band can be expressed as

$$
\left|\psi_{\mathrm{FB}}\right\rangle=(2 \Omega)^{-1 / 2} \sum_{j=1}^{N} \zeta_{j}\left(a_{j}^{\dagger}-c_{j}^{\dagger}\right)|\mathrm{vac}\rangle .
$$

The renormalization coefficient is $\Omega=\sum_{j=1}^{N}\left|\zeta_{j}\right|^{2}$ and $\zeta_{j}$ is an arbitrary number. Antisymmetric excitation is diffractionless with a constant intensity [67], different from that in a nonHermitian flat band entirely constituted by EPs $[68,69]$.

When $J=0$ or $\Phi=n \pi+\pi / 2(n \in \mathbb{Z}), H_{k}$ is chiral symmetric $\left(\mathrm{CH}_{k} \mathcal{C}^{-1}=-H_{k}\right)$ and supports a zero energy flat band; the eigenfunction of $H_{k}$ for $\Phi=\pi / 2$ is given by

$$
f_{k}=[1, i(J-\gamma) /(v+r \cos k),-1]^{\mathrm{T}} .
$$

The flat band states are CLSs [83], as will be discussed in the next section. In a Hermitian lattice $(\gamma=0)$, the flat band appears in the absence of asymmetric coupling; $E_{\mathrm{FB}}=0$ when $J=0$ [Fig. 2(a)] or $E_{\mathrm{FB}}=(-1)^{n+1} J$ when $\Phi=n \pi(n \in \mathbb{Z})$. The bands experience a conical intersection for $J=0$, similar to that in the Lieb lattice $[84,85]$. 


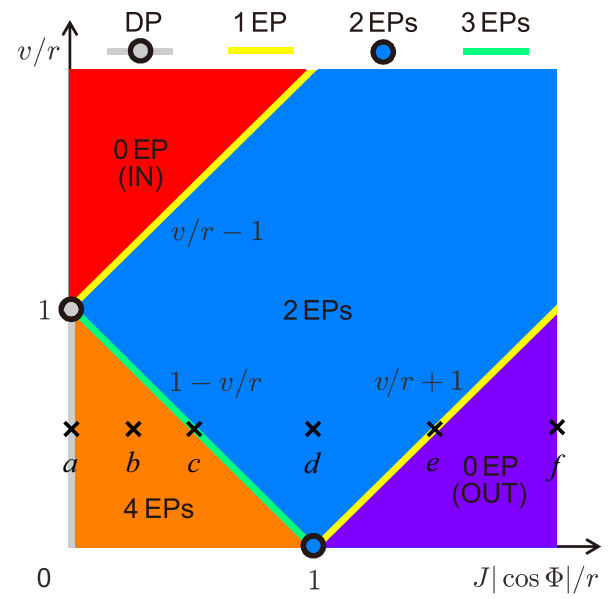

FIG. 3. Phase diagram of the flat band for $\Phi \neq n \pi+\pi / 2$ $(n \in \mathbb{Z})$. The energy bands for the system at parameters marked by points $a-f$ are depicted in Figs. 2(a)-2(f), respectively.

Figure 2 depicts the energy bands of the system at various intracell couplings when the flat band is formed. The intracell couplings expand the energy bands; the flat band energy depends on the intracell coupling $J$, but is independent of the intracell coupling $v$ [Eq. (9)]. Thus the flat band appears in the band gap of the dispersive bands at a strong coupling $v$, and the flat band is the lowest-energy band outside the dispersive bands at a strong coupling $J$. The intercell crossstitch coupling $r=0$ is a trivial case for the existence of flat band; thus we choose $r$ as the unit to demonstrate the influence of the intracell couplings $v$ and $J$ on the energy bands. Figure 3 is the phase diagram of the flat band in the parameter space of intracell couplings, where the phases are classified by the number of EPs in the flat band embedded in the continuum. The boundaries $v / r-1$ and $1-v / r$ indicate that the band touching occurs at $k=\pi$, while the boundary $v / r+1$ indicates that the band touching occurs at $k=0$. Typical cases of the energy bands are depicted in Fig. 2, which reflects the relation between the flat band and dispersive bands; the representative energy bands in the red region of Fig. 3 are depicted in Fig. 4(b) for $v=2$ (zero EP).

For $J=0$, the lattice system is chiral symmetric. A zeroenergy flat band appears, the other two dispersive bands are symmetric, and $E_{k}\left[E_{k}^{2}-2(v+r \cos k)^{2}+\gamma^{2}\right]=0$. The intracell coupling $v$ generates three energy bands, and the band gaps are proportional to this coupling; the intercell coupling $r$ induces dispersion of the upper and lower energy bands. The dispersive bands are gapped when the intercell coupling is weak, that is, $v / r>1$. A dispersive band each locates above and below the zero-energy flat band (belongs to the red region of Fig. 3). At a strong intercell coupling $v / r \leqslant 1$, the coupling $v+r \cos k$ in $H_{k}$ can vanish; thus the band gaps vanish and two dispersive bands touch at $\left|k_{\mathrm{DP}}\right|=\arccos (-v / r)$ in the Brillouin zone (the gray line in Fig. 3). The band touching points are diabolic points of twofold Hermitian degeneracy, which move from $\pi / 2$ toward $\pi$ as the intracell coupling $v$ increases from zero to $r$. The energy bands tighten under the influence of gain and loss [86]; this can be seen in Fig. 4 by comparing Figs. 4(a), 4(b), and 4(c). Without the band gap $(v / r \leqslant 1)$, the energy bands are vulnerable to non-Hermiticity; any nonzero $\gamma$ brings the system into a $\mathcal{P} \mathcal{T}$-symmetry broken phase and the eigenenergy starts to become complex around $k_{\mathrm{DP}}$. The band gaps at $v / r>1$ protect the system by rendering it robust against a certain degree of non-Hermiticity $\gamma=\sqrt{2}(v-r)$, where the band gaps vanish at $\left|k_{\mathrm{EP}}\right|=\pi$. In the presence of non-Hermiticity, the gapless band touching points are EPs, where both the real and imaginary parts of the spectrum are gapless.

For $J \neq 0$, the flat band energy shifts from zero and intersects a dispersive band at the EPs

$$
\left(v+r \cos k_{\mathrm{EP}}\right)^{2}=J^{2} \cos ^{2} \Phi .
$$

This condition is useful for understanding the phase diagram (Fig. 3) and is obtained as follows. If the system supports a flat band, Eq. (5) reduces to $E_{k}^{3}-\left(2 s_{k}^{2}+J^{2} \cos ^{2} \Phi\right) E_{k}-$ $2 s_{k}^{2} J \cos \Phi=0$. At the band touching point, two among the three roots of $E_{k}$ are equal; thus $\left(2 s_{k}^{2}+J^{2} \cos ^{2} \Phi\right)^{3} / 3^{3}-$ $\left(s_{k}^{2} J \cos \Phi\right)^{2}=0$ should be satisfied. After simplification, we obtain $\left(8 s_{k}^{2}+J^{2} \cos ^{2} \Phi\right)\left(s_{k}^{2}-J^{2} \cos ^{2} \Phi\right)^{2}=0$; then, the condition Eq. (13) for the band intersection EPs is acquired. The number of EPs is determined from the competitions between the coupling strengths as shown in Fig. 3. At weak intracell couplings $v, J$, four EPs are obtained from $v+r$ $\cos k_{\mathrm{EP}}= \pm J \cos \Phi$; at moderate intracell couplings $v, J$, two EPs are obtained from $v+r \cos k_{\mathrm{EP}}=+J \cos \Phi(v+$ $\left.r \cos k_{\mathrm{EP}}=-J \cos \Phi\right)$; and EP disappears in the situation that either the intracell coupling $v$ or $J$ is very strong $\left[k_{\mathrm{EP}}\right.$ has no real solution in Eq. (13)], which corresponds to the case that the flat band appears isolatedly inside or outside the dispersive bands with zero EP, respectively.

In the orange and cyan regions of Fig. 3, the band gap diminishes as the degree of non-Hermiticity increases and the flat band appears when the band gap closes at $\gamma=\gamma_{\mathrm{FB}}$ [67]. Therefore, the flat band intersects the dispersive band and becomes the bound states in the continuum $[87,88] . \gamma_{\mathrm{FB}}$ is the $\mathcal{P} \mathcal{T}$-symmetric phase transition point of the non-Hermitian lattice. In the red (violet) region of Fig. 3, the flat band at $\gamma=\gamma_{\mathrm{FB}}$ is isolated inside (outside) the dispersive bands with band gaps, where EP does not exist. The boundary between the gapped red (violet) phase and the gapless blue phase with two EPs is $v-r(v+r)$, where two EPs merge to one at the edges (center) of the Brillouin zone at $\left|k_{\mathrm{EP}}\right|=\pi$ (0). The boundaries of distinct phases in the phase diagram indicate that the band gap vanishes for stronger critical nonHermiticity at $\gamma_{\mathrm{c},-}\left(\gamma_{\mathrm{c},+}\right)$ with

$$
\gamma_{\mathrm{c}, \pm}=\sqrt{2(v \pm r)^{2}+J^{2}-3 \sqrt[3]{(v \pm r)^{4} J^{2} \cos ^{2} \Phi}}
$$

where $\gamma_{\mathrm{c}, \pm}$ is obtained from the cubic equation of the energy bands of $H_{k}$, that is, $\operatorname{det}\left(H_{k}-E_{k} I_{3 \times 3}\right)=0$. The energy bands of $H_{k}$ are no longer entirely real when the band gap vanishes. We can directly verify that $\gamma_{\mathrm{c}, \pm}>\gamma_{\mathrm{FB}}$ in the phases where the energy bands are gapped.

The intracell couplings $J$ and $v$ expand the energy bands and the band gap widths, and the intercell coupling $r$ induces dispersion. Thus the relation between energy bands varies with the variation in the competition of system couplings. The flat band may appear inside [Fig. 4(b) (zero EP)], at the 

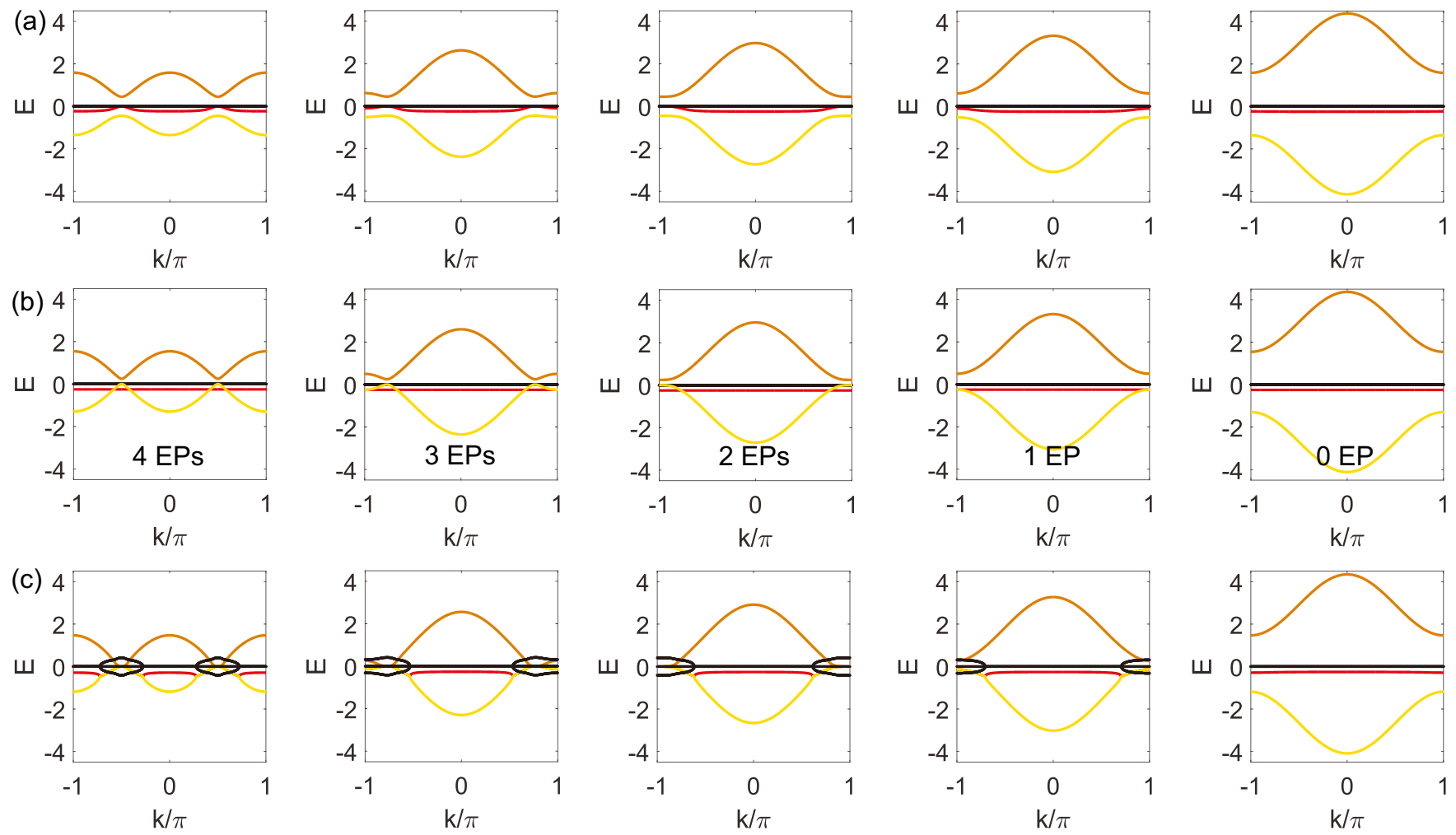

FIG. 4. Complex band structures at various $v$ and $\gamma$ values for $J=1 / 2, \Phi=\pi / 3$, and $r=1$. (a) $\gamma=\gamma_{\mathrm{FB}} / 2$, (b) $\gamma=\gamma_{\mathrm{FB}}$, and (c) $\gamma=$ $3 \gamma_{\mathrm{FB}} / 2$ for $v=0,3 / 4,1,5 / 4$, and 2 from the left to the right panels, respectively. As $v$ increases in (b), the flat band energy is unchanged, but the dispersive bands expand; thus the band intersections reduce from four EPs to zero EP. $k=-\pi$ and $k=\pi$ represent an identical point.

intersection [Figs. 2(a)-2(e)], or outside [Fig. 2(f)] the dispersive bands. At a strong intracell coupling, $J|\cos \Phi|>v+r$, the flat band appears outside the dispersive bands [Fig. 2(f)]. At a moderate intracell coupling, $|v-r|<J|\cos \Phi|<v+r$, the flat band intersects the lower dispersive band and two EP2s (two-state coalescence) appear at $k_{\mathrm{EP}}$ [Fig. 2(d)]. When $J|\cos \Phi|<r-v$, the flat band intersects the lower dispersive band and four EP2s appear at $k_{\mathrm{EP}}$ [Fig. 2(b)]; moreover, with no band gap at $\gamma=0$ [Fig. 2(a)], the non-Hermiticity brings two dispersive bands closer and the two band touching points split into four EP2s. When EP2s appear at the center $\left(k_{\mathrm{EP}}=0\right)$ and the edges $\left(k_{\mathrm{EP}}= \pm \pi\right)$ of the Brillouin zone, pairs of EP2s merge and leave three EP2s [Fig. 2(c)], two EP2s [Fig. 2(d)], and one EP2 [Fig. 2(e)]. At a weak intracell coupling, $J|\cos \Phi|<v-r$, the dispersive bands are gapped and the flat band appears inside [Fig. 4(b) (zero EP)].

When the system is under chiral symmetry at $J=0$ or $\Phi=n \pi+\pi / 2(n \in \mathbb{Z})$, the EPs in the flat band are EP3 (three-state coalescence). Two EP3s $\left|k_{\mathrm{EP}}\right|=\arccos (-v / r)$ exist in the flat band for $v<r$, they merge to one EP3 at $|k|=\pi$ for $v=r$, and EP3 disappears for $v>r$. All the EPs can be determined from $\Delta_{k}=27 s_{k}^{4} J^{2} \cos ^{2} \Phi-\left[\left(2 s_{k}^{2}+J^{2}-\right.\right.$ $\left.\gamma^{2}\right]^{3}=0$.

The flat band is partially flat except for $\gamma=\gamma_{\mathrm{FB}}$, and the flatness is lost near $k_{\mathrm{EP}}$ and increases as $\gamma$ approaches $\gamma_{\mathrm{FB}}$. When $\gamma<\gamma_{\mathrm{FB}}$, the lattice system is in the exact $\mathcal{P} \mathcal{T}$ symmetric phase, and the energy bands become closer as non-Hermiticity increases. The flat band formed at the $\mathcal{P} \mathcal{T}$ symmetric phase transition point $\gamma=\gamma_{\mathrm{FB}}$, is entirely flat with the bands touching and band gaps closing. When $\gamma>\gamma_{\mathrm{FB}}$, the system is in the broken $\mathcal{P} \mathcal{T}$-symmetric phase, the flatness is most robust around the center of the Brillouin zone and is severely lost at the edges of the Brillouin zone. In Fig. 4, the energy bands of $H_{k}$ are depicted for parameters $J=$ $1 / 2, \Phi=\pi / 3$, and $r=1$ at different $v$ and $\gamma$ values. At a weak non-Hermiticity $\gamma<\gamma_{\mathrm{FB}}$, the energy bands are gapped [Fig. 4(a)]; at an appropriate non-Hermiticity $\gamma=\gamma_{\mathrm{FB}}$, the flat band appears [Fig. 4(b)]; at a strong non-Hermiticity $\gamma>\gamma_{\mathrm{FB}}$, the system is in the broken $\mathcal{P} \mathcal{T}$-symmetric phase [54] and the energy bands are complex [Fig. 4(c)]. Figures 4(a)-4(c) show the energy bands of $H_{k}$ for $\gamma=\gamma_{\mathrm{FB}} / 2, \gamma_{\mathrm{FB}}$, and $3 \gamma_{\mathrm{FB}} / 2$, respectively, at $v$ varying from zero to 2 . The bands expand as the intracell coupling $v$ increases and shrink as $\gamma$ increases. At a weak intracell coupling, $v<r-J|\cos \Phi|$, where four EPs appear at critical $\gamma_{\mathrm{FB}}$, the two lower bands form two windows separated by the four EPs at large non-Hermiticity and the central band near $|k|=0, \pi$ is nearly flat; at a moderate intracell coupling, $|r-J| \cos \Phi||<v<r+J|\cos \Phi|$, where two EPs appear at critical $\gamma_{\mathrm{FB}}$, the partial flat band near $|k|=$ $\pi$ vanishes, and the central band near $k=0$ is nearly flat, whose flatness increases as $v$ increases. The flat band appears in the band gap between the dispersive bands for a strong intracell coupling $v>r+J|\cos \Phi|$, where EP disappears. The bifurcation of the energy bands at the EPs significantly destroys the band flatness. A nearly flat region appears away from the EPs, shrinks, and finally vanishes with increasing non-Hermiticity; subsequently, $H_{k}$ has one real band and two conjugate pure imaginary bands. 


\section{COMPACT LOCALIZED STATES}

The flat band energy is insensitive to the boundary conditions. At $\Phi=n \pi+\pi / 2(n \in \mathbb{Z})$, the lattice is chiral symmetric and has a zero-energy flat band. The flat band CLSs are confined in three unit cells; only the edge modes are confined in two unit cells under an open boundary condition. The non-Hermitian lattice has two pairs of edge modes that are localized at two boundaries of the lattice and $N-4$ confined modes that are localized inside the lattice. They are formed through destructive interference at the central sublattice $B$.

The eigenfunctions of the upper and lower sublattices $A$ and $C$ should satisfy $\psi_{A_{j}}+\psi_{C_{j}}=0$. The subscript in the wave functions $\psi$ indicates the site number. Note that sites $A_{j}$ and $C_{j}$ have an identical coupling $v$ to $B_{j}$ in the $j$ th unit cell and identical couplings $r / 2$ to $B_{j \pm 1}$ in the neighbor unit cells; thus the contributions from $A_{j}$ and $C_{j}$ cancel each other and vanish in the Schrödinger equations of $B_{j}$ and $B_{j \pm 1}$. Consequently, the steady-state Schrödinger equations for the sublattice $B$ are satisfied because $\psi_{A_{j}}+\psi_{C_{j}}=0$ and due to the zero energy of the flat band, that is, $0=E_{\mathrm{FB}} \psi_{B_{j}}=v\left(\psi_{A_{j}}+\psi_{C_{j}}\right)+$ $(r / 2)\left(\psi_{A_{j-1}}+\psi_{C_{j-1}}\right)+(r / 2)\left(\psi_{A_{j+1}}+\psi_{C_{j+1}}\right)$.

If the eigenfunction $\psi_{B_{j}}=0, B_{j}$ and $A_{j+1}, C_{j+1}$ are effectively decoupled; moreover, the interference of $A_{j}$ and $C_{j}$ is destructive at $B_{j+1}$. Therefore, $B_{j}=0$ results in a decoupling between the $j$ th and $(j+1)$ th unit cells, forming a state that is localized on the left side of the $j$ th unit cell (similarly, a state that is localized on the right side of the $j$ th unit cell is formed with the decoupling of $B_{j}$ and $A_{j-1}, C_{j-1}$ ). Specifically, $\psi_{B_{1}}=0$ is possible when $\gamma=J$, which is the critical non-Hermiticity $\gamma_{\mathrm{FB}}$ for $\Phi=n \pi+\pi / 2(n \in \mathbb{Z})$. This leads to the decoupling of the whole sublattice $B$ from the lattice system $H$ and the eigenfunction being antisymmetric and independent of the lattice size. $\psi_{B_{2}}=0$ corresponds to a state confined in the first two unit cells. The Schrödinger equations for the lattice system consisting of two unit cells under an open boundary condition are

$$
\begin{gathered}
i \dot{\psi}_{A_{1}}=i \gamma \psi_{A_{1}}+v \psi_{B_{1}}+J e^{i \Phi} \psi_{C_{1}}+\frac{r}{2} \psi_{B_{2}}, \\
i \dot{\psi}_{B_{1}}=v\left(\psi_{A_{1}}+\psi_{C_{1}}\right)+\frac{r}{2}\left(\psi_{A_{2}}+\psi_{C_{2}}\right), \\
i \dot{\psi}_{C_{1}}=-i \gamma \psi_{C_{1}}+v \psi_{B_{1}}+J e^{-i \Phi} \psi_{A_{1}}+\frac{r}{2} \psi_{B_{2}}, \\
i \dot{\psi}_{A_{2}}=i \gamma \psi_{A_{2}}+v \psi_{B_{2}}+J e^{i \Phi} \psi_{C_{2}}+\frac{r}{2} \psi_{B_{1}}, \\
i \dot{\psi}_{B_{2}}=v\left(\psi_{A_{2}}+\psi_{C_{2}}\right)+\frac{r}{2}\left(\psi_{A_{1}}+\psi_{C_{1}}\right), \\
i \dot{\psi}_{C_{2}}=-i \gamma \psi_{C_{2}}+v \psi_{B_{2}}+J e^{-i \Phi} \psi_{A_{2}}+\frac{r}{2} \psi_{B_{1}} .
\end{gathered}
$$

The non-normalized zero mode eigenfunction is $[\varphi, \phi]^{\mathrm{T}}$ as illustrated in Fig. 5(a), where $\varphi \equiv\left(\psi_{A_{1}}, \psi_{B_{1}}, \psi_{C_{1}}\right)=$ $(1, i J / v-i \gamma / v,-1) \quad$ and $\quad \phi \equiv\left(\psi_{A_{2}}, \psi_{B_{2}}, \psi_{C_{2}}\right)=$ $[r /(2 v), 0,-r /(2 v)]$.

The energy-band structure shown in Fig. 5(a) only appears at the lattice boundary, $[\varphi, \phi]^{\mathrm{T}}$, which is the zero mode for a lattice with more unit cells connected at the right side of the two-unit-cell lattice because $\psi_{B_{2}}=0$ (i.e., the zero mode of the extended lattice is unoccupied on the additional unit cells). The structure's mirror reflection corresponds to the zero
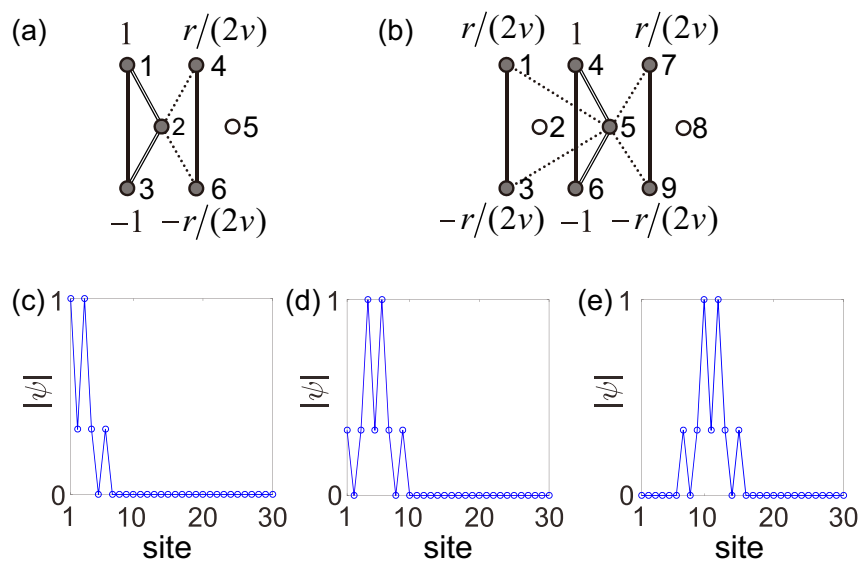

FIG. 5. Schematic of destructive interference for the lattices consisting of (a) two and (b) three unit cells under an open boundary condition. The couplings connected to the unoccupied passive sublattice (hollow circles) are hidden. The wave function of the central site $B_{j}$ is $i(J-\gamma) / v ; A_{j}$ and $C_{j}$ are marked at the top and bottom of the schematic. The occupied sites are represented by gray circles. (c),(d) Edge mode; (e) inner confined mode. The parameters are $\Phi=\pi / 2, J=1, r=1, v=3 / 2$, and $\gamma=1 / 2$.

mode for a lattice with its left side capable of connecting more unit cells. $\psi_{B_{1}}=\psi_{B_{3}}=0$ corresponds to a state that is confined in the first three unit cells $[\phi, \varphi, \phi]^{\mathrm{T}}$, as illustrated in Fig. 5(b). The structure shown in Fig. 5(b) can be formed inside the lattice $H$ with more than three unit cells; $[\phi, \varphi, \phi]^{\mathrm{T}}$ characterizes the probability distributions of the zero mode for a lattice with additional unit cells connected at both the left and right sides.

Under an open boundary condition, $H$ has two pairs of edge modes $\psi_{\mathrm{EMi}}, \mathcal{P} \mathcal{T} \psi_{\mathrm{EMi}}$ in the form

$$
\psi_{\mathrm{EMi}}=[\varphi, \phi, \ldots]^{\mathrm{T}},
$$

and $\psi_{\mathrm{EMii}}, \mathcal{P} \mathcal{T} \psi_{\mathrm{EMii}}$ in the form

$$
\psi_{\mathrm{EMii}}=[\phi, \varphi, \phi, \ldots]^{\mathrm{T}} .
$$

The edge modes only distribute in the two or three unit cells at the lattice boundaries; ... in Eqs. (21) and (22) represents the unit cells $\left(A_{j}, B_{j}, C_{j}\right)$ with vanishing occupation $(0,0,0)$. The edge modes in Eqs. (21) and (22) are depicted in Figs. 5(c) and $5(\mathrm{~d})$, respectively.

The inner lattice CLSs are in the form

$$
\psi_{\mathrm{CLS}}=[\ldots, \phi, \varphi, \phi, \ldots]^{\mathrm{T}},
$$

which is independent of the boundary conditions. The confined modes distribute in the three unit cells that are localized inside the lattice with the passive sublattice $B$ at the edges of the three unit cells being unoccupied. The $\mathcal{P} \mathcal{T}$-symmetric counterpart $\mathcal{P} \mathcal{T} \psi_{\text {CLS }}$ belongs to the confined modes. The confined modes for the unoccupied first two and last five unit cells are depicted in Fig. 5(e). All zero modes are degenerate, and their superpositions are also zero modes. The trapping mechanism is destructive interference assisted by the synthetic magnetic flux inside the unit cells [10,23,24]. Each pair of edge modes [Eqs. (21) and (22)] can form one pair 

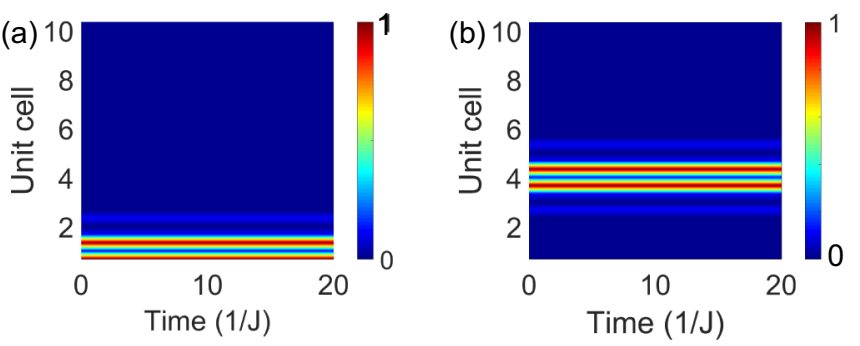

FIG. 6. Time evolution of initial excitations. The intensity $|\psi(t)|^{2}$ is depicted. The parameters are $\Phi=\pi / 2, J=1, r=$ $1, v=3 / 2$, and $\gamma=1 / 2$, which are identical to the parameters in Fig. 5.

of $\mathcal{P} \mathcal{T}$-symmetric zero modes; the confined modes [Eq. (23)] can form another $N-4 \mathcal{P} \mathcal{T}$-symmetric zero mode.

Any zero mode excitation is confined inside that lattice without escaping and its dynamics exhibits no diffraction effect. This is numerically simulated in a lattice of size $N=$ 30 with 10 unit cells. The dynamics for the excitation of the zero edge mode confined in two unit cells [Fig. 5(c)] is depicted in Fig. 6(a). The zero mode is confined at the lattice boundary without spreading or escaping. The dynamics of the excitation of an inner lattice that confines the zero mode [Fig. 5(e)] is depicted in Fig. 6(b). The excitation of any superposition of CLSs is localized and diffractionless in the dynamical process. This dynamics is considerably different from that of a confined polynomial increase of excitation in the non-Hermitian lattices, where flat bands are entirely constituted by EPs $[68,69]$.

Equations (21)-(23) are valid for the zero-energy flat band when $J=0$. For the lattice without chiral symmetry, that is, $\Phi \neq n \pi+\pi / 2(n \in \mathbb{Z})$, the eigenfunction of the central sublattice $B$ is zero for the flat band $E=E_{\mathrm{FB}}$ at $\gamma=\gamma_{\mathrm{FB}}$ under both periodical and open boundary conditions. The flat band is constituted by the CLSs that are confined in the single unit cell. Its eigenfunctions are given by Eqs. (21)-(23) with $\varphi$ replaced by $\varphi=(1,0,-1)$ and $\phi$ unchanged; the eigenfunctions change into the superposition of antisymmetric excitations in the neighboring single unit cells.

\section{SUMMARY}

In this work, we first report a configuration that supports a flat band due to the destructive interference at an appropriate match between the synthetic magnetic flux and nonHermiticity. The flat band energy is flexible at different appropriate matches instead of pinning to zero, and the flat band can intersect the dispersive bands or appear isolatedly inside or outside the dispersive band gap; the flat band states form bound states in the continuum when energy bands intersect.

A quasi-1D $\mathcal{P} \mathcal{T}$-symmetric non-Hermitian lattice is proposed, whose unit cell is a triangular lattice enclosed synthetic magnetic flux. The synthetic magnetic flux is attributed to the AB-type nonreciprocal phase factor in the intracell coupling. We demonstrated the flat band induced by the interplay of synthetic magnetic flux and non-Hermiticity. The phase diagram characterizes the relation between the flat band and dispersive bands. When the flat band intersects the dispersive band, the flat band appears at the $\mathcal{P} \mathcal{T}$-symmetric phase transition point. The eigenfunctions of the flat band are compact localized states, which are confined within a few unit cells inside or at the edges of the non-Hermitian lattice.

Our findings provide insights into the interplay of synthetic magnetic flux and non-Hermiticity in the application of controllable flat band and bound states in the continuum in nonHermitian metamaterials. Further studies of the AharonovBohm cage, nonreciprocal localization, and anomalous edge modes would be interesting in both theoretical and experimental aspects.

\section{ACKNOWLEDGMENTS}

This work was supported by NSFC (Grant No. 11605094).
[1] D. Guzmán-Silva, C. Mejía-Cortés, M. A. Bandres, M. C. Rechtsman, S. Weimann, S. Nolte, M. Segev, A. Szameit, and R. A. Vicencio, New J. Phys. 16, 063061 (2014).

[2] D. Leykam, S. Flach, O. Bahat-Treidel, and A. S. Desyatnikov, Phys. Rev. B 88, 224203 (2013).

[3] L. Ge, Ann. Phys. (Berlin) 529, 1600182 (2017).

[4] F. Baboux, L. Ge, T. Jacqmin, M. Biondi, E. Galopin, A. Lemaître, L. Le Gratiet, I. Sagnes, S. Schmidt, H. E. Türeci, A. Amo, and J. Bloch, Phys. Rev. Lett. 116, 066402 (2016).

[5] E. H. Lieb, Phys. Rev. Lett. 62, 1201 (1989).

[6] V. Apaja, M. Hyrkás, and M. Manninen, Phys. Rev. A 82, 041402 (2010).

[7] R. A. Vicencio, C. Cantillano, L. Morales-Inostroza, B. Real, C. Mejía-Cortés, S. Weimann, A. Szameit, and M. I. Molina, Phys. Rev. Lett. 114, 245503 (2015).

[8] S. Mukherjee, A. Spracklen, D. Choudhury, N. Goldman, P. Öhberg, E. Andersson, and R. R. Thomson, Phys. Rev. Lett. 114, 245504 (2015).
[9] S. Xia, A. Ramachandran, S. Xia, D. Li, X. Liu, L. Tang, Y. Hu, D. Song, J. Xu, D. Leykam, S. Flach, and Z. Chen, Phys. Rev. Lett. 121, 263902 (2018).

[10] C. E. Creffield and G. Platero, Phys. Rev. Lett. 105, 086804 (2010).

[11] A. Bermudez, T. Schaetz, and D. Porras, Phys. Rev. Lett. 107, 150501 (2011).

[12] N. Masumoto, N. Y. Kim, T. Byrnes, K. Kusudo, A. Lö ffler, S. Höfling, A. Forchel, and Y. Yamamoto, New J. Phys. 14, 065002 (2012).

[13] G.-W. Chern, C.-C. Chien, and M. Di Ventra, Phys. Rev. A 90, 013609 (2014).

[14] C. Wu, D. Bergman, L. Balents, and S. Das Sarma, Phys. Rev. Lett. 99, 070401 (2007).

[15] T. Jacqmin, I. Carusotto, I. Sagnes, M. Abbarchi, D. D. Solnyshkov, G. Malpuech, E. Galopin, A. Lemaître, J. Bloch, and A. Amo, Phys. Rev. Lett. 112, 116402 (2014).

[16] J. T. Chalker, T. S. Pickles, and P. Shukla, Phys. Rev. B 82, 104209 (2010). 
[17] P. Shukla, Phys. Rev. B 98, 054206 (2018).

[18] J. Vidal, R. Mosseri, and B. Douçot, Phys. Rev. Lett. 81, 5888 (1998).

[19] A. R. Kolovsky, A. Ramachandran, and S. Flach, Phys. Rev. B 97, 045120 (2018).

[20] A. Ramachandran, A. Andreanov, and S. Flach, Phys. Rev. B 96, 161104(R) (2017).

[21] D. Leykam, A. Andreanov, and S. Flach, Adv. Phys. X 3, 1473052 (2018).

[22] P. W. Anderson, Phys. Rev. 109, 1492 (1958).

[23] J. Vidal, B. Doucot, R. Mosseri, and P. Butaud, Phys. Rev. Lett. 85, 3906 (2000).

[24] S. Longhi, Opt. Lett. 39, 5892 (2014).

[25] S. Mukherjee and R. R. Thomson, Opt. Lett. 40, 5443 (2015); 42, 2243 (2017).

[26] S. Mukherjee, M. Di Liberto, P. Öhberg, R. R. Thomson, and N. Goldman, Phys. Rev. Lett. 121, 075502 (2018).

[27] M. Kremer, I. Petrides, E. Meyer, M. Heinrich, O. Zilberberg, and A. Szameit, arXiv:1805.05209.

[28] C. M. Bender and S. Boettcher, Phys. Rev. Lett. 80, 5243 (1998).

[29] C. Dembowski, B. Dietz, H.-D. Gräf, H. L. Harney, A. Heine, W. D. Heiss, and A. Richter, Phys. Rev. E 69, 056216 (2004).

[30] A. Ruschhaupt, F. Delgado, and J. G. Muga, J. Phys. A 38, L171 (2005).

[31] S. Klaiman, U. Günther, and N. Moiseyev, Phys. Rev. Lett. 101, 080402 (2008).

[32] R. El-Ganainy, K. G. Makris, D. N. Christodoulides, and Z. H. Musslimani, Opt. Lett. 32, 2632 (2007); K. G. Makris, R. El-Ganainy, D. N. Christodoulides, and Z. H. Musslimani, Phys. Rev. Lett. 100, 103904 (2008); Z. H. Musslimani, K. G. Makris, R. El-Ganainy, and D. N. Christodoulides, ibid. 100, 030402 (2008).

[33] A. Guo, G. J. Salamo, D. Duchesne, R. Morandotti, M. VolatierRavat, V. Aimez, G. A. Siviloglou, and D. N. Christodoulides, Phys. Rev. Lett. 103, 093902 (2009).

[34] O. Bendix, R. Fleischmann, T. Kottos, and B. Shapiro, Phys. Rev. Lett. 103, 030402 (2009).

[35] L. Jin and Z. Song, Phys. Rev. A 80, 052107 (2009); 81, 032109 (2010).

[36] Y. N. Joglekar, D. Scott, M. Babbey, and A. Saxena, Phys. Rev. A 82, 030103(R) (2010).

[37] C. E. Rüter, K. G. Makris, R. El-Ganainy, D. N. Christodoulides, M. Segev, and D. Kip, Nat. Phys. 6, 192 (2010).

[38] X. Luo, J. Huang, H. Zhong, X. Qin, Q. Xie, Y. S. Kivshar, and C. Lee, Phys. Rev. Lett. 110, 243902 (2013).

[39] H. Schomerus, Phys. Rev. Lett. 104, 233601 (2010); S. Malzard, C. Poli, and H. Schomerus, ibid. 115, 200402 (2015).

[40] J. M. Zeuner, M. C. Rechtsman, Y. Plotnik, Y. Lumer, S. Nolte, M. S. Rudner, M. Segev, and A. Szameit, Phys. Rev. Lett. 115, 040402 (2015).

[41] H. Jing, S. K. Özdemir, X.-Y. Lü, J. Zhang, L. Yang, and F. Nori, Phys. Rev. Lett. 113, 053604 (2014).

[42] B. Peng, S. K. Özdemir, F. Lei, F. Monifi, M. Gianfreda, G. L. Long, S. Fan, F. Nori, C. M. Bender, and L. Yang, Nat. Phys. 10, 394 (2014).

[43] L. Chang, X. Jiang, S. Hua, C. Yang, J. Wen, L. Jiang, G. Li, G. Wang, and M. Xiao, Nat. Photon. 8, 524 (2014).
[44] X. Zhu, H. Ramezani, C. Shi, J. Zhu, and X. Zhang, Phys. Rev. X 4, 031042 (2014).

[45] R. Fleury, D. Sounas, and A. Alù, Nat. Commun. 6, 5905 (2015).

[46] B. He, L. Yang, Z. Zhang, and M. Xiao, Phys. Rev. A 91, 033830 (2015); B. He, L. Yang, and M. Xiao, ibid. 94, 031802(R) (2016).

[47] Z. Zhang, Y. Zhang, J. Sheng, L. Yang, M.-A. Miri, D. N. Christodoulides, B. He, Y. Zhang, and M. Xiao, Phys. Rev. Lett. 117, 123601 (2016).

[48] K. Ding, G. Ma, M. Xiao, Z. Q. Zhang, and C. T. Chan, Phys. Rev. X 6, 021007 (2016).

[49] A. Cerjan, A. Raman, and S. Fan, Phys. Rev. Lett. 116, 203902 (2016); A. Cerjan and S. Fan, ibid. 118, 253902 (2017).

[50] L. Feng, R. El-Ganainy, and L. Ge, Nat. Photon. 11, 752 (2017).

[51] Y. Kominis, T. Bountis, and S. Flach, Phys. Rev. A 95, 063832 (2017).

[52] D. L. Sounas and A. Alù, Nat. Photon. 11, 774 (2017).

[53] S. Longhi, Y. Kominis, and V. Kovanis, Europhys. Lett. 122, 14004 (2018).

[54] R. El-Ganainy, K. G. Makris, M. Khajavikhan, Z. H. Musslimani, S. Rotter, and D. N. Christodoulides, Nat. Phys. 14, 11 (2018).

[55] Y. D. Chong, L. Ge, H. Cao, and A. D. Stone, Phys. Rev. Lett. 105, 053901 (2010); W. Wan, Y. Chong, L. Ge, H. Noh, A. D. Stone, and H. Cao, Science 331, 889 (2011); Y. Sun, W. Tan, H.-Q. Li, J. Li, and H. Chen, Phys. Rev. Lett. 112, 143903 (2014).

[56] L. Feng, Y.-L. Xu, W. S. Fegadolli, M.-H. Lu, J. E. B. Oliveira, V. R. Almeida, Y.-F. Chen, and A. Scherer, Nat. Mater. 12, 108 (2013).

[57] B. He, L. Yang, X. Jiang, and M. Xiao, Phys. Rev. Lett. 120, 203904 (2018).

[58] L. Jin and Z. Song, Phys. Rev. Lett. 121, 073901 (2018).

[59] H. Xu, D. Mason, L. Jiang, and J. G. E. Harris, Nature (London) 537, 80 (2016).

[60] S. Assawaworrarit, X. Yu, and S. Fan, Nature (London) 546, 387 (2017).

[61] L. Feng, Z. J. Wong, R.-M. Ma, Y. Wang, and X. Zhang, Science 346, 972 (2014).

[62] H. Hodaei, M.-A. Miri, M. Heinrich, D. N. Christodoulides, and M. Khajavikhan, Science 346, 975 (2014).

[63] B. Peng, S. K. Özdemir, M. Liertzer, W. Chen, J. Kramer, H. Yilmaz, J. Wiersig, S. Rotter, and L. Yang, Proc. Natl. Acad. Sci. USA 113, 6845 (2016).

[64] G. Harari, M. A. Bandres, Y. Lumer, M. C. Rechtsman, Y. D. Chong, M. Khajavikhan, D. N. Christodoulides, and M. Segev, Science 359, eaar4003 (2018); M. A. Bandres, S. Wittek, G. Harari, M. Parto, J. Ren, M. Segev, D. Christodoulides, and M. Khajavikhan, ibid. 359, eaar4005 (2018).

[65] M. I. Molina, Phys. Rev. A 92, 063813 (2015).

[66] L. Ge, Phys. Rev. A 92, 052103 (2015).

[67] H. Ramezani, Phys. Rev. A 96, 011802(R) (2017).

[68] D. Leykam, S. Flach, and Y. D. Chong, Phys. Rev. B 96, 064305 (2017).

[69] L. Ge, Photon. Res. 6, A10 (2018).

[70] B. Qi, L. Zhang, and L. Ge, Phys. Rev. Lett. 120, 093901 (2018). 
[71] J. D. Bodyfelt, D. Leykam, C. Danieli, X. Yu, and S. Flach, Phys. Rev. Lett. 113, 236403 (2014).

[72] S. Flach, D. Leykam, J. D. Bodyfelt, P. Matthies, and A. S. Desyatnikov, Europhys. Lett. 105, 30001 (2014).

[73] C. Gneiting, Z. Li, and F. Nori, Phys. Rev. B 98, 134203 (2018).

[74] T. Ramos, B. Vermersch, P. Hauke, H. Pichler, and P. Zoller, Phys. Rev. A 93, 062104 (2016); P. Lodahl, S. Mahmoodian, S. Stobbe, A. Rauschenbeutel, P. Schneeweiss, J. Volz, H. Pichler, and P. Zoller, Nature (London) 541, 473 (2017).

[75] Z. Yu and S. Fan, Nat. Photon. 3, 91 (2009); K. Fang, Z. Yu, and S. Fan, Phys. Rev. Lett. 108, 153901 (2012); P. Roushan, C. Neill, A. Megrant, Y. Chen, R. Babbush, R. Barends, B. Campbell, Z. Chen, B. Chiaro, A. Dunsworth, A. Fowler, E. Jeffrey, J. Kelly, E. Lucero, J. Mutus, P. J. J. O’Malley, M. Neeley, C. Quintana, D. Sank, A. Vainsencher, J. Wenner, T. White, E. Kapit, H. Neven, and J. Martinis, Nat. Phys. 13, 146 (2017).

[76] E. Li, B. J. Eggleton, K. Fang, and S. Fan, Nat. Commun. 5, 3225 (2014).

[77] M. Hafezi, Int. J. Mod. Phys. B 28, 1441002 (2014).

[78] M. Aidelsburger, M. Atala, S. Nascimbène, S. Trotzky, Y.-A. Chen, and I. Bloch, Phys. Rev. Lett. 107, 255301 (2011).
[79] W. Zheng and H. Zhai, Phys. Rev. A 89, 061603(R) (2014); N. H. Lindner, G. Refael, and V. Galitski, Nat. Phys. 7, 490 (2011); N. Goldman and J. Dalibard, Phys. Rev. X 4, 031027 (2014).

[80] N. Goldman, J. C. Budich, and P. Zoller, Nat. Phys. 12, 639 (2016).

[81] K. M. Sliwa, M. Hatridge, A. Narla, S. Shankar, L. Frunzio, R. J. Schoelkopf, and M. H. Devoret, Phys. Rev. X 5, 041020 (2015).

[82] R. Fleury, D. L. Sounas, C. F. Sieck, M. R. Haberman, and A. Alù, Science 343, 516 (2014).

[83] W. Maimaiti, A. Andreanov, H. C. Park, O. Gendelman, and S. Flach, Phys. Rev. B 95, 115135 (2017).

[84] A. Julku, S. Peotta, T. I. Vanhala, D.-H. Kim, and P. Törmä, Phys. Rev. Lett. 117, 045303 (2016).

[85] D. Leykam and A. S. Desyatnikov, Adv. Phys. X 1, 101 (2016).

[86] A. Szameit, M. C. Rechtsman, O. Bahat-Treidel, and M. Segev, Phys. Rev. A 84, 021806(R) (2011).

[87] Y.-X. Xiao, G. Ma, Z.-Q. Zhang, and C. T. Chan, Phys. Rev. Lett. 118, 166803 (2017).

[88] C. W. Hsu, B. Zhen, A. D. Stone, J. D. Joannopoulos, and M. Soljačić, Nat. Rev. Mater. 1, 16048 (2016). 